\section{Brain, Behavior and Fvolution}

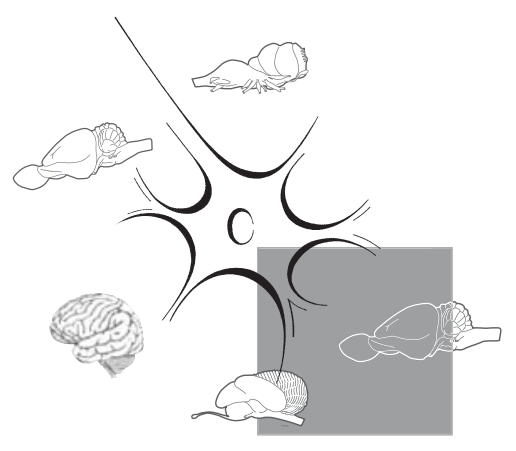

'Evodevo' is an acronym from the words 'evolution and development' that is used to name the field of life sciences focused on the study of differences and similarities in the developmental process of extant species with the aim of understanding how they have evolved from common ancestors. The 'evodevo' research field has expanded greatly over the last century thanks to an increase in the number of research groups focusing their efforts in this discipline and the appearance of science journals specifically devoted to publishing new advances in the field (e.g. Evolution and Development) or journals from a variety of disciplines that publish a considerable percentage of studies that use the 'evodevo' approach (e.g. Brain, Behavior and Evolution).

'Evodevo' researchers use a comparative approach to study a specific developmental process in different but related species to understand the evolutionary process and try to identify primitive and derived characters, homologies, parallelisms or phenomena of convergent evolution. Here, using as an example recent data [CornidePetronio et al., 2011; Hawthorne et al., 2011; Kuscha et al., 2011] on the ability of different vertebrate species to regenerate

Highlights and Perspectives on Evolutionary Neuroscience

Brain Behav Evol 2012;79:1-3

Published online: December 6, 2011 DOI: $10.1159 / 000334187$

\title{
'Evorego': Studying Regeneration to Understand Evolution, the Case of the Serotonergic System
}

\section{Antón Barreiro-Iglesias}

Shriners Hospitals Pediatric Research Center (Center for Neural Repair and Rehabilitation), Temple University School of Medicine, Philadelphia, Pa., USA; Department of Cell Biology and Ecology, CIBUS, Faculty of Biology, University of Santiago de Compostela, Santiago de Compostela, Spain

their serotonergic system after injury in the central nervous system (CNS), I would like to introduce the concept of 'evorego'. In this case, 'evorego' refers to the idea of how we could also try to understand evolution by studying the differences and similarities in the regenerative ability between extant species.

In mammals, including humans, injury to the adult CNS usually causes an irreversible loss of function because of the inability of the mature CNS to regenerate. One of the main causes for the absence of recovery is the inability of injured axons to regenerate and reconnect with their appropriate targets. By contrast, teleost fishes and lampreys are able to regain functionality after injury in the mature CNS. For example, both zebrafish and lampreys are able to recover locomotion that appears normal after a complete spinal cord transection [Becker and Becker, 2008; Barreiro-Iglesias and Rodicio, 2011].

Recent studies have shown that serotonergic neurons have an impressive regenerative ability after spinal cord injury in both zebrafish [Kuscha et al., 2011] and lampreys [Cornide-Petronio et al., 2011]. Using a double labeling tract-tracing tech- nique combined with serotonin immunofluorescence, Cornide-Petronio et al. [2011] reported that axotomized brainstem serotonergic descending axons of lampreys regenerate spontaneously after a complete spinal cord transection and that these axons reinnervate levels below the injury site. The study by Kuscha et al. [2011] has also shown that in adult zebrafish, serotonergic fibers partially reinnervate levels below the site of injury after a complete spinal cord transection and that the degree of reinnervation can be correlated with the degree of functional recovery. Nonetheless, a complete restoration of the serotonergic innervation below the level of injury never occurs in zebrafish and lampreys, even when the animals have regained normal locomotion.

Results in lampreys and zebrafish suggest that phenomena of plasticity and reorganization of the spinal circuits after injury help to achieve the recovery of function in these animals compensating for the incomplete regeneration of injured serotonergic axons. The reason why not all axotomized serotonergic axons regenerate through the site of injury in these animals is not yet known. An intriguing hypothesis is that it

\section{KARGER}

Fax +41613061234 E-Mail karger@karger.ch www.karger.com
Antón Barreiro-Iglesias

Shriners Hospitals Pediatric Research Center (Center for Neural Repair and

Rehabilitation), Temple University School of Medicine

3500 North Broad Street, Philadelphia, PA 19140 (USA)

Tel. +1 215926 9328, E-Mail anton.barreiro.iglesias@ temple.edu 


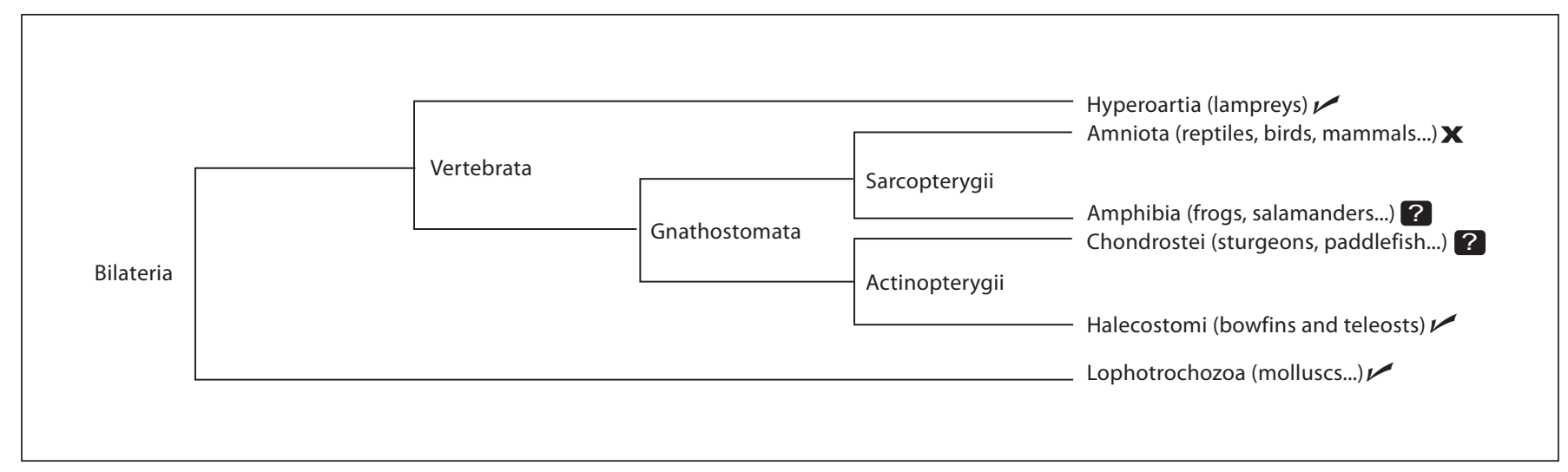

Fig. 1. Phylogenetic tree showing representative groups of bilateria in which the phenomenon of spontaneous regeneration of serotonergic axons after injury has been reported to occur $(\boldsymbol{})$ or not $(\mathbf{X})$. Other key groups of vertebrata in which the phenomenon has not been studied were also included (indicated by question marks). The tree was constructed based on the data deposited in the 'Tree of life web project' (Maddison and Schulz, 2007). Note that not all groups of bilateria were included in the tree.

could be attributed to intrinsic factors of the serotonergic neurons since the extrinsic environment seems to be favorable for axonal regrowth.

The phylogenetic tree depicted in figure 1 indicates species in which the phenomenon of spontaneous regeneration of axotomized serotonergic axons has been observed. Earlier studies reported the spontaneous regeneration of axotomized serotonergic neurons in molluscs [Murphy et al., 1985; Chiasson et al., 1994; Koert et al., 2001] (fig. 1). However, the recent studies in lampreys and zebrafish are the first to show this phenomenon in vertebrate species. Although more studies are needed in other groups of bilateria, especially groups belonging to Ecdysozoa like Arthropoda or Nematoda, the current available data suggest that the ability to regenerate axotomized serotonergic axons in the mature nervous system may be an ancient character of bilateria. Given the data on molluscs, it is more parsimonious to argue that the ability of lampreys and zebrafish to regenerate axotomized serotonergic axons is due to shared ancestry than independent evolution. The findings in lampreys and zebrafish are in marked contrast with the situation in mammals since spontaneous regeneration of serotonergic axotomized axons through the site of injury does not occur in these animals after a complete spinal cord transection [Deumens et al., 2005] (fig. 1). More studies in other groups of vertebrata (e.g. Amphibia, Chondrostei or Diapsida) are needed to determine when this character was lost along the line leading to mammals.

Remarkable sprouting of serotonergic axons has been reported in adult zebrafish at levels above the spinal cord site of injury before the axons start to regenerate and reinnervate levels below the injury site [Kuscha et al., 2011]. Profuse sprouting and branching of numerous serotonergic axons has been also observed at the site of spinal cord injury in the freshwater turtle Trachemys dorbignyi [Rehermann et al., 2009]. Interestingly, the recent study from Silver's group has shown that after a thermocoagulatory lesion in the rat frontoparietal cortex, serotonergic axons persist within the lesion edge and, by the third week post-injury, sprout robustly into the lesion cavity, whereas callosal axons become dystrophic and die back [Hawthorne et al., 2011]. This study confirms previous reports showing that serotonergic axons in mammals also have a great ability to sprout after different types of injury, e.g. chemical lesion in the brain [Zhou et al., 1995] and spinal cord injury [Inman and Steward, 2003; Camand et al., 2004]. Comparison of the current available data suggests that the impressive ability of serotonergic axons to persist and sprout at the site of injury is ancestral for vertebrates and that it was conserved during the evolutionary process leading to mammals, although they lost the ability to fully regenerate these axons.

These recent studies serve as good example of an 'evorego' approach and open up very interesting questions, such as (1) 'What was the evolutionary or selective pressure that favored the loss of regenerative ability in the mammalian CNS during evolution?', (2) 'Is there any selective disadvantage in presence of a high regenerative ability in the adult nervous system?' and (3) 'Was the loss of regenerative ability a selected adaptative trait or was it an epiphenomenon?'.

The use of 'evorego' animal models like lampreys or zebrafish may help to address these and other questions, as well as provide new clues to understand the evolution of regeneration. In addition, studying how these animals are able to regenerate their CNS after injury at mature stages of development could provide important information to propose new therapies for patients with CNS injuries.

\section{Acknowledgments}

A.B.-I. was supported by a Shriners Hospitals Postdoctoral Research Fellowship. Grant sponsor: Xunta de Galicia, Consellería de Economía e Industria (INCITE08PXIB200063PR). 
Becker CG, Becker T (2008): Adult zebrafish as a model for successful central nervous system regeneration. Restor Neurol Neurosci 26:7180.

Barreiro-Iglesias A, Rodicio MC (2011): Lampreys as animal models in neurobiology. SciTopics. http://www.scitopics.com/Lampreys_as_ animal_models_in_neurobiology.html (accessed August 19, 2011).

Camand E, Morel MP, Faissner A, Sotelo C, Dusart I (2004): Long-term changes in the molecular composition of the glial scar and progressive increase of serotoninergic fibre sprouting after hemisection of the mouse spinal cord. Eur J Neurosci 20:1161-1176.

Chiasson BJ, Baker MW, Croll RP (1994): Morphological changes and functional recovery following axotomy of a serotonergic cerebrobuccal neurone in the land snail Achatina $f u$ lica. J Exp Biol 192:147-167.
Cornide-Petronio ME, Ruiz MS, Barreiro-Iglesias A, Rodicio MC (2011): Spontaneous regeneration of the serotonergic descending innervation after spinal cord injury in the sea lamprey. J Neurotrauma DOI: 10.1089/neu.2011. $\underline{1766}$

Deumens R, Koopmans GC, Joosten EA (2005): Regeneration of descending axon tracts after spinal cord injury. Prog Neurobiol 77:57-89.

Hawthorne AL, Hu H, Kundu B, Steinmetz MP, Wylie CJ, Deneris ES, Silver J (2011): The unusual response of serotonergic neurons after CNS injury: lack of axonal dieback and enhanced sprouting within the inhibitory environment of the glial scar. J Neurosci 31:56055616.

Inman DM, Steward O (2003): Ascending sensory, but not other long-tract axons, regenerate into the connective tissue matrix that forms at the site of a spinal cord injury in mice. J Comp Neurol 462:431-449.

Koert CE, Spencer GE, van Minnen J, Li KW, Geraerts WP, Syed NI, Smit AB, van Kesteren RE (2001): Functional implications of neurotransmitter expression during axonal regeneration: serotonin, but not peptides, autoregulate axon growth of an identified central neuron. J Neurosci 21:5597-5606.
Kuscha V, Barreiro-Iglesias A, Becker CG, Becker T (2011): Plasticity of tyrosine hydroxylase and serotonergic systems in the regenerating spinal cord of adult zebrafish. J Comp Neurol DOI: $10.1002 /$ cne.22739.

Maddison DR, Schulz KS (eds) (2007): The tree of life web project. http://tolweb.org.

Murphy AD, Barker DL, Loring JF, Kater SB (1985): Sprouting and functional regeneration of an identified serotonergic neuron following axotomy. J Neurobiol 16:137-151.

Rehermann MI, Marichal N, Russo RE, TrujilloCenóz O (2009): Neural reconnection in the transected spinal cord of the freshwater turtle Trachemys dorbignyi. J Comp Neurol 515: 197-214.

Zhou FC, Azmitia EC, Bledsoe S (1995): Rapid serotonergic fiber sprouting in response to ibotenic acid lesion in the striatum and hippocampus. Brain Res Dev Brain Res 84:89-98. 